\title{
„Lernt vom Genossen Lei Feng!“ oder Selbstopfer und Heldentum in Zeiten des Umbruchs
}

\author{
Dominik Pietzcker
}

\author{
„Ich war ein Held, mein Rubm gewaltig \\ In meinen Bannern rauschten die vier Winde \\ Wenn meine Trommeln lärmten schreieg das Volk \\ Ich habe mein Leben vertan" \\ - Heiner Müller ${ }^{1}$ -
}

\section{Einleitung: Das dichotomische Wagnis}

Die kulturelle Konzeption des Heldentums ist ohne einen kämpferischen Grundton undenkbar, besteht doch das Wesen des Heroischen gerade in der tätigen, durchaus auch kriegerischen, Auseinandersetzung mit der vorgefundenen Wirklichkeit. Die existentielle Zuspitzung liegt präzise in der Dichotomie von Sieg oder Niederlage, Leben oder Tod, im Wagnis also, nur diese beiden Kategorien überhaupt gelten zu lassen. ${ }^{2}$ Wie auch immer die Konsequenz einer solchen Entscheidung ausfällt, in jedem Fall bedarf sie der Notwendigkeit des physischen oder moralischen Kampfes. Ohne diese unausweichliche, also tragische Notwendigkeit, bleibt der bean geste des Heroischen sinnlos und blutleer, ein rein ästhetischer Akt, der das Absurde streift. ${ }^{3}$

Heldentum ist kein historisches Faktum, sondern ein fluider und ironiefähiger Begriff. ${ }^{4}$ Zwischen der antiken (archaischen) und modernen, der europäischen

1 Heiner Müller: Gedichte 1949-1989, Berlin 2007, S. 31 (Nach Po Chü I).

2 Libertad o Muerte war bekanntlich das Losungswort der republikanischen Truppen im Spanischen Bürgerkrieg. Der Held wirft das eigene Leben in die Waagschale der vermeintlich guten oder gar göttlichen Sache. Diese Bereitschaft zur Selbstopferung ist bereits als devotio für die römische Antike verbürgt, vgl. Livius: 8, 9-10. Der Feldherr Publius Decius Mus weiht in der Schlacht am Vesuv (340 v. Chr.) den Göttern sein eigenes Leben und das seiner Feinde, um den Sieg für die eigenen Waffen herbeizuführen: Legiones auxiliaque hostium mecum Deis Manibus Tellurique devoveo. Zur konventionellen Nähe zwischen Held und Tod vgl. auch die bekannten Zeilen in Rilkes Sechster Duineser Elegie (sog. Helden-Elegie, Rainer Maria Rilke: Die Gedichte, Frankfurt am Main 1987, S. 650-651): „Wunderlich nah ist der Held doch den jugendlich Toten. Dauern / ficht ihn nicht an. Sein Anfang ist Dasein; beständig / nimmt er sich fort und tritt ins veränderte Sternbild / seiner steten Gefahr." Man könnte fast sagen, die Entscheidung des Helden ist im Kern stets die zwischen Krieg und Frieden.

3 Als Ausdruck einer solchen heroischen Geste ohne Bedeutung, eines leeren, rein ästhetischen Aktes der Wirklichkeitsverweigerung, kann der rituelle Selbstmord Yukio Mishimas am 25. November 1970 in Tokyo gelten.

4 Die ironische Brechung bereits bei Cervantes Don Quichote (1615) und Lermontow Ein Held unserer Zeit (1840). Zur Literarisierung des Helden vgl. die epochale Studie von 
und asiatischen Vorstellung des Heroischen - und seiner Kritik bzw. Problematisierung - liegen buchstäblich Welten. Am Beispiel des chinesischen Rotarmisten Lei Feng soll aufgezeigt werden, wie das Konzept der heroischen Selbstaufopferung bis hin zur letzten Konsequenz ausagiert und auf breiter Front als ideologisches Vehikel der Massenkommunikation genutzt wird. Sein Lebenslauf, aber auch dessen offizielle Instrumentalisierung, sind ein genuin chinesisches Kulturphänomen, das sich nicht ohne weiteres an ein europäisches Werteverständnis adaptieren lässt. Bevor Lei Feng als Protagonist jedoch genauer betrachtet wird, sei das Leitmotiv des Heroischen aus individueller und kollektiver, östlicher und westlicher Perspektive zumindest im Ansatz ausgeleuchtet. Nur so lässt sich die Sinnhaftigkeit einer exemplarischen chinesischen Heldenvita angemessen einordnen.

\section{Das heroische Selbstverständnis in West und Ost}

Entscheidendes Merkmal des heroischen Selbstverständnisses ist die prinzipielle Opfer-, Leidens- und Entbehrungsbereitschaft des Protagonisten. Jeder Held - jede Heldin ${ }^{5}$ - ist ein Abenteurer, ein Normenbrecher und -überschreiter, der die vermeintliche Sicherheit und Planbarkeit einer geregelten Lebensweise für sich ablehnt. Das Verhältnis des Heroen zur Macht, zu den herrschenden und tonangebenden Minderheiten, ihren jeweiligen Wertvorstellungen und Gesellschaftsentwürfen, ist keinesfalls eindeutig. Es gibt den affirmativen Helden und den oppositionellen, den Helden als Anführer aus der Mitte der Gesellschaft ebenso wie den Helden als Deserteur, Rebell und Aufbegehrender. Für Baudelaire - les litanies de Satan ${ }^{6}$ - ist selbst Luzifer ein Held, für Genet der Verbrecher, ja Mörder. Held ist, wer aus eigener Entscheidung bereit ist, gesellschaftliche Normen vorbildhaft zu erfüllen oder aber sie vollständig zu brechen. Heroen sind demnach entweder Exponenten elitärer Macht- und Moralvorstellungen, die sie affirmativ zu verstärken oder aber als Aufbegehrende zu zerstören suchen. ${ }^{7}$

Lei Feng, der chinesische Repräsentant des Kollektivs, seiner Tugenden und Leitbilder, verkörpert eine Form des Heroismus, die westlich-individualistischen Vorstellungen zu widersprechen scheint. Lei Feng ist das Volk und geht in ihm auf. Wenn er die Massen überragt, so nur, um diese zu erhöhen. Dieser kollektivistische Blickwinkel auf die Figur des Heros entspricht konfuzianischen Denktraditionen, nicht aber europäischen. Ohne Frage ist der Mythos Lei Feng das

Michail M. Bachtin: Autor und Held in der ästhetischen Tätigkeit, Frankfurt am Main 2008, v. a. S. 63-76.

5 Wenn hier und im Folgenden von Helden die Rede ist, so sind damit stets auch Heldinnen gemeint; es sei denn, es handelt sich um konkrete historische oder literarische Personen.

6 Charles Baudelaire: CEuvres complètes I \& II, texte établi, présenté et annoté par Claude Pichois, Paris 1975, Bd. 1, S. 123.

7 „Le vrai héros s'amuse tout seul“, bemerkt zwar Baudelaire (ebd., S. 682), doch bleibt der Held als Einzelgänger und Außenseiter historisch die Ausnahme. 
Produkt einer interessegeleiteten politischen Medialisierung; dennoch wird an seinem gelebten Beispiel auch eine prinzipielle kulturelle Differenz zwischen China und Europa sichtbar.

\section{Individuum und Kollektiv im Bürgerkrieg}

Der revolutionäre Held, zu dem auch Lei Feng gehört, ist eine Geburt der staatlichen Krise und des Bürgerkrieges. Dieser fordert in chaotischer Permanenz ein lebensgefährliches Einstehen für die eigenen Überzeugungen; dabei verspricht er keinen Ruhm und noch nicht einmal den Sieg. Das prägende Merkmal des Bürgerkrieges besteht vielmehr darin, dass er die Position des unbeteiligten Dritten nicht vorsieht und die gesamte Bevölkerung unter sich zwingt. Von einer kollektivistischen Warte aus gesehen ist der Bürgerkrieg wirklich der große Würger, der nicht von einem Einzelnen niedergerungen werden kann, sondern nur von den zu einer disziplinierten Einheit geformten Massen einer „Volksarmee“8. So mutiert die individuelle und einsam gedachte Figur des Helden, der die Vielen vermeintlich überragt und sich gerade nicht zu ihnen zählt, ${ }^{9}$ zum Repräsentanten, genauer gesagt zur Verkörperung, des größtmöglichen gemeinsamen Nenners, des ,Volkes'. Gerade diese Repräsentativität des Einzelnen für die Volksmassen ist die typisch chinesischkonfuzianische Perspektive auf das Phänomen des Helden.

Die treibende Kraft des Heros nach abendländischem Verständnis ist der Gedanke des Wettbewerbs in der ultimativen Überwindung des selbstgewählten Feindes (competitive excellence). Im konfuzianischen Sinne ist hingegen derjenige ein Vorbild, dem es gelingt, die eigenen persönlichen Belange und vitalen Bedürfnisse vollständig mit denen einer überpersönlichen Gruppe in Einklang zu bringen (cooperative values). ${ }^{10}$ „Die Erfordernisse eines Teiles sind den Erfordernissen des Ganzen unterzuordnen". ${ }^{11}$ Dieser Widerspruch der Perspektiven lässt sich jedoch

8 „Gibt es keine Volksarmee, dann gibt es nichts für das Volk. [...] Diese Armee ist dadurch stark, dass alle, die ihr beigetreten sind, bewusst Disziplin halten; sie haben sich vereinigt und kämpfen nicht um Privatinteressen, sondern für die Interessen der breiten Volksmassen und für die Interessen der gesamten Nation. Fest an der Seite des chinesischen Volkes zu stehen und ihm mit ganzem Herzen zu dienen, ist das einzige Ziel dieser Armee." (Mao Zedong: Worte des Vorsitzenden Mao Tsetung, Essen 1967, S. 118-119). Vgl. ebd., 105: „Die stärkste Kraftquelle für die Kriegführung liegt in den Volksmassen.“

9 Achill, Inbegriff des abendländischen Kriegers, kämpft aus persönlicher Rachsucht um Patroklos und nicht zum höheren Ruhme der Achaier (Homer: Ilias, XVIII. Gesang, 9094). Zitiert nach der Übersetzung von Johann Heinrich Voss, nach dem Text der Erstausgabe mit einem Nachwort von Wolf Hartmut Friedrich, Düsseldorf/Zürich 1996.

10 Zur Unterscheidung von kompetitiven und kooperativen Werten (competitive excellence und cooperative values) vgl. Arthur William Hope Adkins: Merit and Responsibility. A Study in Greek Values, Oxford 1960, und Maria Ossowska: Das ritterliche Ethos und seine Spielarten (Reihe Denken und Wissen - Eine polnische Bibliothek), Frankfurt am Main 2007, S. $28 \mathrm{ff} .$, S. 44.

11 Vgl. Zedong: Worte des Vorsitzenden (Anm. 8), S. 287. 
in einem Punkt auflösen. Beide Sichtweisen eint die Bereitschaft des Einzelnen, den ultimativen Preis für die jeweils internalisierte Wertehierarchie zu entrichten.

Das höchste Risiko bleibt, über Geschichte und unterschiedliche Gesellschaftssysteme hinweg, der Verlust des eigenen Lebens. Dieses Risiko ist in einem bevölkerungsreichen Land, in dem notorisch Hunger, Missernten, Krieg und Okkupation durch fremde Mächte herrschen, wie im China der 1920er bis Ende der 1940er Jahre, deutlich höher als etwa in Zürich oder Stockholm zur gleichen Zeit. Die Notwendigkeit, hohe und höchste Lebensrisiken auf sich nehmen zu müssen, ist also keineswegs ein Akt der Freiwilligkeit; wohl aber die Art und Weise, wie diese angenommen oder eben abgelehnt werden. Die bekannte amerikanische Journalistin Agnes Smedley, die in den 1930er Jahren aus China über den Krieg der kommunistischen Truppen und der Guomintang (GMD) gegen die japanischen Invasionsstreitkräfte berichtete, bemerkt lapidar: „The interaction between environment and will power shapes character. "12 Je härter und gefährlicher das jeweilige gesellschaftliche Umfeld, desto radikaler handeln die ihm ausgesetzten Individuen.

\section{Vom adligen zum proletarischen Helden}

Die polnische Soziologin Maria Ossowska erwähnt in ihrer klassischen Studie über das ritterliche Ethos vier Beschäftigungen, welche denjenigen, die an der Spitze der gesellschaftlichen Hierarchie stehen, zur Ehre gereichen, nämlich die Ausübung der Herrschaft, religiöse Zeremonien, Kriegführen und Sport. „Die Bedingungen für die Entstehung einer adeligen Kultur sind [...] Besitz, Grundeigentum und Tradition." ${ }^{13}$ Was aber bleibt jenen zu tun übrig, die nicht oder nur unzulänglich über diese Eigentumstitel verfügen?

Vormoderne Helden ${ }^{14}$ stammten aus einer sakralisierten Kriegerkaste, die nur in Ausnahmefällen, etwa in revolutionären Situationen oder zu Bürgerkriegszeiten, Aufstiegsmöglichkeiten für Außenseiter bot. Machtfragen und daraus resultierende kriegerische Handlungen wurden von bewaffneten Minderheiten ausgefochten. Leitragender dieser Auseinandersetzungen war ausnahmslos die Zivilbevölkerung, die daher besonderer Schutzrechte bedurfte. Historisch eskalierte der Krieg im 20. Jahrhundert vom regional begrenzten Konflikt zur kontinentalen und globalen Auseinandersetzung. Die grauenhaften Verwundungen und Verstümmelungen, Werk der modernen industriellen Zerstörungsmaschinerie, taten ein Übriges, um die romantisch-reaktionär-aristokratischen Vorstellungen eines ritterlichen Waffenganges vollständig zu diskreditieren. Der pazifistische Bildband Krieg dem Kriege von Friedrich Ernst, der die menschlichen Verwüstungen schonungslos darstellte,

12 Agnes Smedley zit. nach: Janice R. / Stephen R. MacKinnon: Agnes Smedley. The Life and Times of an American Radical, London 1988, S. 2.

13 Ossowska: Das ritterliche Ethos (Anm. 10), S. 35.

14 Zu diesem Begriff vgl. Maurice Bowra: Heroic Poetry, London 1952. 
gehörte zu den großen Bucherfolgen der Weimarer Republik. Schon aus den Schützengräben des Ersten Weltkrieges hatte der englische Dichter Wilfried Owen gefragt und keine Antwort gefunden: „What passing bells of these who die as cattle?"15 Der kriegerische Held als Herausforderer und Bändiger des eigenen Schicksals hatte endgültig ausgedient.

Historisch war die Zeit damit reif für einen neuen Typus des tradierten Heros, den Typus des Arbeiterhelden. Er ist der Repräsentant des Volkes, dem, in den Worten Ernst Jüngers „eine elementare Beziehung zum Kriege gegeben ist und der sich deshalb kriegerisch aus eigenen Mitteln zu vertreten vermag". ${ }^{16}$ Die neuen vitalen Helden stammen aus der Mitte des Volkes und lösen die obsolete Kriegerelite ab das ist schon die Schlusspointe bei Jean Renoirs epochalem Schwarzweißfilm La Grande Illusion (1936). Im Aufkommen eines neuen Heldentypus, des kämpfenden Arbeiters, spiegelt sich die ideologische Zeitenwende seit 1917. Überall, in Europa wie in Asien, streben kommunistisch-revolutionäre Organisationen an die Macht, die den Umsturz der tradierten Werte und ihrer gesellschaftlichen Repräsentation mit Waffengewalt und gestützt auf die anonymen Massen des Volkes einfordern. In Russland (1917) und später in China (1949) gelingt die Revolution. Mit ihr setzt sich das ideologische Credo der Apotheose der Arbeiter- und Bauernschaft als gesellschaftliches Erlösungsversprechen durch:

Was ist Arbeit? Arbeit bedeutet Kampf. An diesen Orten gibt es Schwierigkeiten und Probleme, zu deren Überwindung bzw. Lösung wir benötigt werden. Wir gehen dorthin zur Arbeit und zum Kampf, um die Schwierigkeiten zu überwinden. ${ }^{17}$

Der ideologisch überhöhte Arbeiter, buchstäblich: der „Proletarier aller Länder“ (Marx), repräsentiert die soziologische Schicht der Zukunft, welche berufen ist, die Atavismen des bürgerlichen Zeitalters zu überwinden. Diese eschatologische Annahme, ob irrtümlich oder nicht, bleibt auch für die Konzeption des Heros nicht folgenlos. Denn der Arbeiter als Held ist eben nicht Teil einer dem Volk enthobenen Auslese der Besten - dies wäre ja das Grundprinzip aller aristokratischen Gesellschaften -, sondern repräsentiert die Tugenden seines Herkunftsmilieus, ohne sich über dieses zu erheben:

Die Arbeiterschaft hat etwas zu bieten, das dem Kapital [...] zumindest ebenbürtig, wenn nicht überlegen ist: die Arbeit - die „menschliche Arbeit“. Sie wird verstanden als körperliche Arbeit das heißt als diejenige menschliche Arbeit, die am sinnfälligsten „Arbeit" ist; als produktive Arbeit, das heißt eine unmittelbare wertschaffende Leistung; als

15 Vgl. Benjamin Britten / Wilfred Owen: War Requiem, Freiburg 1993, S. 7. Der italienische Historiker Enzo Traverso sieht in den Brutalismen des industriellen Krieges eine wesentliche Ursache für die völlig enthemmten Gewaltexzesse des Totalitarismus, vor allem des Nationalsozialismus (Enzo Traverso: Im Banne der Gewalt. Der europäische Bürgerkrieg 1914-1945, München 2008, S. 184-187).

16 Ernst Jünger: Der Arbeiter. Herrschaft und Gestalt, Stuttgart 1981, S. 159.

17 Zedong: Worte des Vorsitzenden (Anm. 8), S. 235. 
primäre Arbeit, das heißt eine Funktion, die für die anderen, für die Gesellschaft, eine fundamentale Voraussetzung ihrer Existenz schafft. ${ }^{18}$

Wie der Arbeiter und der Bauer Kinder des Volkes sind, ist die Volksarmee eine Armee der arbeitenden Massen, die für ihre eigene Emanzipation ins Feld ziehen. Die Motivation, im Letzten für die eigene Freiheit zu kämpfen und sich dadurch auf eine höhere Moral berufen zu können, unterscheidet ideologisch geschulte Kadertruppen von militärischen Einheiten, die nur nach disziplinarischen Prinzipien geführt werden. ${ }^{19}$ Anna L. Strong, amerikanische Reporterin vom chinesischen Kriegsschauplatz in den 1930er und 1940er Jahren, schreibt über die Opferbereitschaft der kommunistischen Truppen: „The Chinese common soldiers were heroic in hand-to-hand combat; whole battalions of them died [...] without quitting their posts. But more than the dying of heroes was needed; for a soldier's job is not to die but to win. ${ }^{\text {"20 }}$ Der Sieg der kommunistischen Truppen im chinesischen Bürgerkrieg war im Kern ein Sieg des Volkes über die den traditionellen Hierarchien und dem Geist des Aristokratismus verpflichtete Kaste der Landeigentümer, Amtsträger (sog. Mandarine) und Fabrikanten. „Hirse und Gewehre sind stärker als Flugzeuge und Tanks" lautete Maos bekannte Devise. ${ }^{21}$ Während Tschang Kai Tschek und die Guomintang die wirtschaftlichen Interessen nationalistischer Grundbesitzer und Industrieller vertraten, konnten die kommunistischen Kommandeure glaubhaft machen, die Interessen der Volksmassen zu geschichtlicher und politischer Wirksamkeit zu führen. Unter der Überschrift Revolutionärer Heroismus schreibt Mao im April 1945: „Tausende und Abertausende von Helden sind uns vorangegangen und haben mutig ihr Leben für die Interessen des Volkes hingegeben. Lasst uns ihre Fahne hochheben und vorwärts schreiten auf dem von ihrem Blut getränkten Weg!“'22 Die Helden des Volkes und die Volksmassen werden auf symbolischer, militärischer und politischer Ebene eins. ${ }^{23}$

\section{Der exemplarische chinesische Held: Leben und früher Tod von Lei Feng}

Als Waisenkind, welches in Partei und Armee eine neue Familie findet, repräsentiert Lei Feng einen neuen heroischen Typus, den Held als Massenvorbild und

18 Heinrich Popitz (Hg.): Das Gesellschaftsbild des Arbeiters. Soziologische Untersuchungen in der Hüttenindustrie, Tübingen 1977, S. 238.

19 „Für wen kämpft ihr?“ lautet sinnigerweise die Überschrift eines kommunistischen Propagandaplakates, welches sich an die Truppen der Guomintang wandte (vgl. Agnes Smedley: China blutet. Vom Sterben des alten China, Moskau/Leningrad 1936, S. 288).

20 Anna L. Strong zit. nach: Agnes Smedley: China Fights Back. An American Woman with the Eighth Route Army, Peking 2003, S. xv.

21 Mao Zedong: Interview mit der amerikanischen Korrespondentin Anna Louise Strong, Peking 1961, S. 10.

22 Ders.: Worte des Vorsitzenden (Anm. 8), S. 214.

$23 \mathrm{Vgl}$. auch die Widmung von Agnes Smedleys Tatsachenbericht Battle Hymn of China aus dem chinesischen Bürgerkrieg (Agnes Smedley: Battle Hymn of China, New York 1943): „To the soldiers of China, poor glorious pioneers [...].“ 
-spiegel, der durch das eigene Handeln seinem Herkunftsmilieu, dem ,einfachen Volk, die moralische Legitimation der Machtausübung verleiht. Die paradoxe Fallhöhe besteht darin, dass der jugendliche Held durch seinen frühen Tod nicht stürzt, sondern umgekehrt, die Mitte des Volkes durch ihn eleviert wird. Der Held aus dem Volk repräsentiert das Volk als Held.

Lei Feng wurde 1940 in der südchinesischen Provinz Hunan als Sohn einer armen Bauernfamilie geboren. Mit sieben Jahren verlor er seine Eltern. Sein Vater wurde von japanischen Truppen getötet, seine Mutter, die von der Familie des Verpächters schikaniert wurde, beging kurz darauf Selbstmord. ${ }^{24} 1960$ trat er in die Volksbefreiungsarmee ein, avancierte schnell zum Truppenführer und wurde Parteimitglied. Das fleißige Studium von Maos Werken lehrte ihn ein einfaches und selbstloses Leben, das ganz der revolutionären Sache ergeben war. Von seinen guten Taten, derentwegen er später Berühmtheit erlangen sollte, machte er wenig Aufheben. Er spendete seine Ersparnisse für Flutopfer, wusch nach langen Märschen die Füße seiner Kameraden und stopfte in der Freizeit ihre Socken. 1961 lud man ihn zu Vorträgen ein, bei denen er über die Beweggründe seines uneigennützigen Handelns Auskunft geben sollte. ${ }^{25}$ Ein Jahr später wurde er bei einem Unfall von einem Holzmast erschlagen. ${ }^{26}$ Ein kurzes, exemplarisches Leben im Dienst einer Idee. Entsprechend aufwändig war das Bemühen von offizieller Seite, Lei Fengs Leben und frühen Tod politisch und ideologisch zu nutzen. „Lernt vom Genossen Lei Feng!“ wurde unmittelbar nach seinem Tod zum geflügelten Wort, wirkungsvoll bis heute. Während der Kulturrevolution wurde Lei Feng nicht weniger sakralisiert als in den Zeiten der wirtschaftlichen Öffnung. Bis in die Gegenwart „findet sich in fast jeder chinesischen Schule ein Porträt Lei Fengs, und die Schulkinder verinnerlichen seinen Geist des Altruismus dadurch, dass sie Passagen aus seinem Tagebuch auswendig lernen“. ${ }^{27} 1997$ wurde ein Film über sein Leben gedreht, es

$24 \mathrm{Zu}$ den biographischen Angaben von Lei Feng vgl. v. a. Michael Ostheimer: Lernen mit Lei Feng, in: Neue Züricher Zeitung, 6. Juni 2003, www.nzz.ch/article8RX5S-1.261575, 28. Dezember 2018, sowie Clement A. Dugue: Lei Feng: China's Evolving Cultural Icon, 1960s to the Present, University of New Orleans Theses and Dissertations, scholarworks.uno.edu/td/996, 20. Dezember 2018 und Andreas Steen: „Im Frühjahr kehrt Lei Feng zurück." Zur gesellschaftlichen und politischen Bedeutung eines Mustersoldaten, in: Das Neue China 34.3, 2007, S. 13-18. Obwohl Lei Feng noch im heutigen China eine volkstümliche Figur der postrevolutionären Ära darstellt, ist er im Westen weitestgehend unbekannt. Die Herkunft aus einfachen und unterdrückten Verhältnissen gehört zur typischen Saga der Revolutionäre der ersten Stunde. So schreibt z. B. auch der Mao-Begleiter Tschen Tschang-Feng über sich (Tschang-Feng Tschen: Mit dem Vorsitzenden Mao auf dem Langen Marsch, Peking 1972, S. 1): „Ich wurde [...] im Dorf Lingnao als Sohn einer sehr armen Bauernfamilie geboren. Als ich elf Jahre alt war, starb meine Mutter. Um die Familie zu ernähren, verdingte sich mein Vater den Grundbesitzern. Unsere Armut und Unterdrückung [...] ließen mich in früher Kindheit schon die räuberische Ausbeuterklasse aus tiefstem Herzen hassen." Lei Feng führte über Jahre ein Tagebuch, dessen Echtheit jedoch kritisch diskutiert wird.

26 Vgl. Ostheimer: Lernen mit Lei Feng (Anm. 24).

27 Vgl. ebd. Vgl. auch den Blog-Eintrag von Si Ying: Lei Feng, My Hero, 7. Januar 2007, myhero.com/Lei_Feng_FIHS_07_ul, 18. Dezember 2018: „Teachers help us learn all sorts 
gibt ein Lei Feng Museum und eine Vielzahl von Plakaten, Publikationen und Zitaten, die den Vorbildcharakter einer kollektiven Heldenvita zum Ausdruck bringen: „Ein Menschenleben ist endlich, aber der Dienst am Menschen ist unendlich“. ${ }^{28}$ Hinzu kommen popkulturelle Merchandising-Artikel, „the popularity of Lei Feng as a revolutionary myth and icon boosts sales of memorabilia, souvenirs, hats and shoes, though probably without much effect on promoting his virtues". 29

Lei Feng symbolisiert eine spezifische Variante des Heroischen, den jugendlichen Helden als Sohn und Protagonisten des Volkes. Das Bild des Heroischen wird kollektiviert, das Einzelcharisma zum „Gruppencharisma“. ${ }^{30}$ Als individuelle Erscheinung erfüllt er alle Voraussetzungen einer kollektivistischen Mythologisierung: niedere Herkunft, tadelloser politischer Werdegang, Fleiß und Aufopferungsbereitschaft sowie, mindestens ebenso wichtig wie alles vorher Genannte zusammen, ein früher Tod, der kategorisch ausschließt, dass ein höheres Lebensalter die absoluten Positionen der eigenen Jugend relativiert: „In einem sozialistischen Staat mit einer größtenteils verarmten und unwissenden Bevölkerung fiel es nicht schwer, die Ideale der Frugalität und Nächstenliebe zum Aufbau des Neuen China anzunehmen. Andererseits waren das Nachahmen und Lernen von [...] Helden und Künstlern seit jeher Bestandteil der konfuzianischen Lernkultur, in welche auch die neuen Vorbilder integriert wurden." ${ }^{31}$

Als Vorbild der Massen überlebte Lei Feng auch die späte Mao-Ära. In modernisierter Form wurde seine Geschichte an die neue Epoche der Marktöffnung unter Deng Xiaoping adaptiert. Nun figuriert Lei Feng als Rollenmodell für Wissbegierde, Lernbereitschaft und Geschäftstüchtigkeit: „Within the domain of the market economy, Lei would not only perform acts of generosity, but he would open businesses and create jobs for his fellow countrymen." 32 Die überraschende Anpassungsfähigkeit seiner Lebensgeschichte an die unterschiedlichen politisch-ökonomischen Strömungen von über fünf Dekaden liegt zweifellos im konfuzianischen Bedeutungskern seiner exemplarischen Heldenvita: „One possible explanation for

of facts about Lei Feng in our childhood, and this study gives us a sense of the kind of selflessness that Lei Feng practiced. It is worthwhile to a person's spirit to study Lei Feng. When I see others in difficulty, I remember Lei Feng and I can also help others to learn about Lei Feng."

28 Lei Feng zit. nach Steen: „Im Frühjahr kehrt Lei Feng zurück“ (Anm. 24).

29 Andreas Steen: To Live is to Serve the People. The Spirit of Model Soldier Lei Feng in Postmodernity, in: Alison Hulme (Hg.): The Changing Landscape of China's Consumerism, Oxford 2014, S. 151-176, hier S. 151.

30 Norbert Elias: Gruppencharisma und Gruppenschande, hg. von Erik Jentgens mit einer biographischen Skizze von Hermann Korte, Marbach a. N. 2014 (1964), greift den Begriff des Gruppencharismas (Max Weber) kritisch auf: „Viele Beobachtungen zeigen ohne Zweifel, dass Gruppencharisma mit einem höheren Grade von Verallgemeinerung vereinbar ist als individuelles Charisma [...]. Aber auch an diesem Punkte wird man fragen müssen, ob es sich dabei wirklich um eine Verallgemeinerung eines ursprünglich heroischen und außergewöhnlichen Charisma handelt.“

31 Steen: „Im Frühjahr kehrt Lei Feng zurück“ (Anm. 24), S. 17.

32 Dugue: Lei Feng (Anm. 24), S. 23. 
the immediate popularity of the Lei Feng model in the 60 s and his tenacious staying power through three decades may rest in the fact that his roots predate the modern period. Although he was a hero designed to promote socialist construction, he displays certain virtues and characteristics that link him with Chinese models of the past. ${ }^{\text {" } 33} \mathrm{Zu}$ den konfuzianischen Tugenden, die in die chinesische Moderne transferiert wurden, gehören die Betonung der Gruppenidentität, die vordergründige Verfolgung von kollektiven, nicht individuellen Interessen, die nicht hinterfragbare Autorität der kommunistischen Idee für das eigene Tun sowie ein an Gemeinschaftswerten orientierter handlungsmotivierender Konformismus.

Ein Land und ein politisches System, denen es gelingt, die enormen intellektuellen und wirtschaftlichen Kräfte zu bündeln, die in einer solchen Mentalität ruhen, werden eine bemerkenswerte und zielgerichtete Dynamik freisetzen. Gerade in seiner Mustergültigkeit verkörpert der „Genosse Lei Feng“ ein System, welches auf der Internalisierung von Regelkonformität und Leistungsbereitschaft beruht. Mit dieser Kombination ist das gegenwärtige China auf dem besten Weg, zum neuen Hegemon der östlichen Hemisphäre aufzusteigen und die westliche Welt nicht nur wirtschaftlich und technologisch, sondern auch geistig und gesellschaftlich herauszufordern. Lei Feng wird damit zum symbolon für Chinas beeindruckende Entwicklung seit 1949. Dies erklärt auch seine anhaltende Popularität, die ohne einen dahinter liegenden politischen Willen undenkbar wäre. ${ }^{34}$ Heute werden Lei Feng, John Lennon und Bruce Lee, alle drei Jahrgang 1940, in einem Atemzug genannt. So in der offiziellen Zeitung Renmin Ribao ${ }^{35}$ - ein offenes Zugeständnis an die global dominierende Popkultur und zugleich der Versuch, tradierte kommunistische Rollenmuster in die Ära des Konsumismus hinüberzuretten.

\section{Die Heroen der Aufbauarbeit: deutsche und chinesische Heldenbilder nach 1945}

Agnes Smedley notiert während des chinesischen Bürgerkrieges in ihr Tagebuch: „Surely, I thought, no people were so gracious, none so magnificent as those willing to face death for the sake of all that they thought good in life. "36 Ohne Frage, das Pathos des Heroischen hat, zumal in der zweiten Hälfte des 20. Jahrhunderts, in China einen völlig anderen Klang als in Deutschland. Die historischen Gründe sind offensichtlich. Während sich das kommunistische China auf den Sieg der ei-

33 Gay Garland Reed: The Lei Feng Phenomenon in the PRC, Dissertation, University of Virginia (Typoskript, Order Nr. 9218751), Virginia 1991, S. 66-67.

34 Bemerkenswerterweise verliert die Figur des Lei Feng in der Millenium-Generation an Vorbildcharakter. Die Redewendung: „Du bist ja wie Lei Feng!“ wird umgangssprachlich zunehmend ironisierend verwendet. Für diesen Hinweis dankt der Autor Frau Shuang Wu, Berlin.

35 Vgl. Mark Siemons: Der gute Mensch Lei Feng. Erinnerung an einen Soldat, in: Frankfurter Allgemeine Zeitung, 22. März 2012, www.faz.net/-gsf-6ym1x, 17. Dezember 2019.

36 Smedley: Battle Hymn of China (Anm. 23), S. 366. 
genen Waffen und die Erfüllung der marxistischen Eschatologie berufen konnte, blickten die zwei deutschen Nachfolgestaaten des Dritten Reiches auf eine vollkommen kompromittierte und moralisch entgleiste Geschichte zurück, die gerade auch vermeintlich noble, konservative und vaterländische Werte und Vorbilder unwiderruflich zerstört hatte. Insbesondere die militärische Tradition des, so das verklärte Ideal, wagemutigen (,schneidigen'), dabei korrekten Offiziers preußischer Prägung, war durch die deutschen Kriegsverbrechen, vor allem in Osteuropa, vollständig gebrochen. Die Wortpaarung Heroismus und Deutschland ist seitdem nationalsozialistisch kontaminiert und politisch unmöglich. Durch die grundsätzlich divergierende ideologische Ausrichtung der beiden deutschen Staaten bis 1989 lassen sich jedoch unterschiedliche Annäherungen an den Komplex des Heroischen und seiner Umformung im Zivilleben (,Courage') nachzeichnen.

Die neuen Helden der jungen Bundesrepublik waren mitnichten die sieglosen Feldmarschälle und traumatisierten Kriegsheimkehrer. Die weitestgehend entpolitisierte Öffentlichkeit suchte ihr säkulares Heil in einem ausschließlich wirtschaftlich motivierten Pragmatismus. Auf die (niemals produzierte), Wunderwaffe' des Dritten Reiches folgte das handfeste ,Wirtschaftswunder' der Adenauer-Ära. Die heilsgeschichtliche Konnotation des,Wunders' kam nicht von ungefähr, barg doch der unerwartete ökonomische Aufschwung auch ein quasireligiöses Erlösungsversprechen. Im Westdeutschland der 1950er Jahre gewinnen der erfolgreiche Geschäftsmann, der - angelsächsischem Berufsbild folgende - Manager, der mächtige Wirtschaftsmagnat an Renommee und Strahlkraft. Die Anstrengungen verlagern sich vom diskreditierten weltanschaulich-militärischen Komplex in den Bereich der freien und sozialen Marktwirtschaft. In der Belletristik hingegen dominieren die geschlagenen Helden eines Borchert, Koeppen oder Böll. Die gängigen Vorstellungen des Heroischen werden entmilitarisiert, ökonomisch umgedeutet oder literarisch ins Selbstquälerische und -zerstörerische gewendet. Ungebrochen kühne und moralisch integre Akteure des realen Lebens wie der Jurist Fritz Bauer und der schwäbische Gewerkschaftsführer Willi Bleicher bleiben insulare Erscheinungen und begründen keinerlei institutionelle Tradition. Ein wie auch immer geartetes heroisches Narrativ ist nirgendwo in der Bundesrepublik zu bemerken, außer vielleicht in der bewusst aus der Zeit gefallenen, sonderbar unbekümmerten Prosa Ernst Jüngers. ${ }^{37}$

Der sozialistische Teil Deutschlands dagegen pflegte mit dem Kult des Antifaschismus und der simplifizierenden Überzeugung, auf der richtigen Seite der Geschichte zu stehen, eine ideologische Überhöhung des Heroischen unter ökonomischen Vorzeichen. Hier war nicht der durchsetzungsstarke erfolgreiche Individualist, sondern bezeichnenderweise der ,Held der Arbeit ${ }^{6}$ gesellschaftliches Vorbild, übrigens nicht nur im Bereich der industriellen Produktion, sondern auch in

37 Auch Gottfried Benns späte Gedichte bezeugen eine gewisse stoische Unbeeindrucktheit. Allerdings ist diese weit entfernt von den Bennschen Positionen der 1930er Jahre, etwa in dem historischen Essay Dorische Welt. 
Kunst und Kultur. ${ }^{38}$ Der Gedanke eines starken Engagements für die Gemeinschaft, bis hin zur Zuspitzung des Selbstopfers, war gemäß der sozialistischen Doktrin nur konsequent. Inspiriert von sowjetischen Vorbildern oder Legenden (propagandistisch macht dies keinen Unterschied) wie dem Bauernjungen Morosow oder dem Rotarmisten Matrossow dominierte die Auffassung vom kollektiven Sinn individueller Handlungen und Entbehrungen. Aufgrund des ideologischen Zerwürfnisses zwischen dem Kommunismus chinesisch-maoistischer Prägung und dem sowjetischen Block in den mittleren 1950er Jahren war in der zeitlichen Nachfolge die Figur Lei Fengs jedoch weder in der Sowjetunion noch in ihren Satellitenstaaten von höherem Interesse. Auch im Westen Deutschlands wurde Lei Feng in seiner gesellschaftlichen Vorbildfunktion erst mit der wirtschaftlichen Öffnung Chinas bekannt, wenn auch eher als kulturwissenschaftliches Kuriosum. ${ }^{39}$

Dabei war Lei Feng nicht das einzige Rollenvorbild für Linientreue und Redlichkeit, Fleiß und Selbstlosigkeit im China der postrevolutionären Industrialisierung. Zu hoher Bekanntheit gelangte auch der Pionier der chinesischen Erdölindustrie, der Bohrarbeiter Wang Jinxi, genannt der „Eiserne Wang“. Gemeinsam mit seinem Team gehörte Wang Jinxi zu den ersten, die das nordchinesische Ö1feld von Daqing, heute eine Millionenstadt, explorierten. Die offizielle Zeitung China Today erinnert an ihn:

Er wurde in einer armen Bauernfamilie geboren. Mit 13 Jahren schuftete er auf einem Ölfeld in Yumen. Nach der Gründung des Neuen China wurde er Leiter eines Bohrteams. Im Jahr 1960 ist er einer Anweisung folgend nach Daqing umgezogen. [...] Nachdem Wang Jinxi in Daqing angekommen war, entlud er mit anderen Arbeitern mit Händen und Schultern die Einzelteile der großen Bohrmaschinen, die 50 bis 60 Tonnen wogen, vom Güterzug. [...] Einmal sprang er in den Bohrschlamm, um das austretende Öl zu stoppen und Verluste zu verhindern. Ein anderes Mal hatte er sieben Tage und Nächte ununterbrochen gearbeitet. Die Einheimischen sagten zu ihm: „Teamleiter Wang, du bist ja ein eiserner Mann." So verbreitete sich der Name Eiserner Mann Wang im ganzen Land, während man seinen wahren Namen vergaß..$^{40}$

Wie Lei Feng weist auch Wang Jinxi alle Elemente einer proletarischen Hagiographie auf. Herkunft aus einfachem Milieu, Härte zu sich selbst, Bereitschaft zu körperlichem Risiko und überdurchschnittlicher Anstrengung sind die wiederkehrenden Leitmotive. Entscheidend für die extreme Handlungsweise ist jedoch ihre Motivation. Ultimatives Ziel ist, ganz in konfuzianischer Tradition, nicht das eigene Fortkommen (in westlichen Kategorien: Beförderung, Gehaltserhöhung, Firmenwagen, Risikozuschlag), sondern das Gemeinwohl. Mit 47 Jahren

38 Neben den Bergleuten Erich Giebner und Josef „Sepp“ Wenig wurden auch Schriftsteller wie Ludwig Renn und Anna Seghers sowie hohe Funktionäre wie der Geheimdienstchef Markus Wolf und der Devisenbeschaffer Alexander Schalck-Golotkowski mit dem Titel „Held der Arbeit“ ausgezeichnet.

39 Vgl. Michael Bonnin: Le retour des héros, in: Perspectives chinoises 34, 1996, S. 10-19, www.persee.fr/doc/perch_1021-9013_1996_num_34_1_19971996, 17. Dezember 2019.

40 N. Shi / C. Sun: Daqing gestern, heute und morgen, www.chinatoday.com.cn/chinaheute/ g2001/chinaheute-5/daqing.htm, 30. Dezember 2018. 
stirbt Wang Jinxi an einem Krebsleiden. Seine Arbeitsbrigade 1205 besteht bis heute.

Für das westliche Europa wäre ein solcher Lebenslauf nicht nur außerordentlich unwahrscheinlich, sondern auch weit davon entfernt, überhaupt erstrebenswert zu sein, fehlt ihm doch als entscheidendes utilitaristisches Ingredienz der Treiber wirtschaftlichen Eigennutzes. Diese Motivlage, euphemistisch als Selbstverwirklichung und Individualismus umschrieben, ist im säkular geprägten Westen zweifellos vorrangig; Abweichungen von diesem Muster können hier allenfalls befremden. Der „Geist des Kapitalismus“ (Max Weber) westlicher Prägung unterscheidet sich in diesem Punkt fundamental von kollektiven Werthaltungen und Handlungsweisen, wie sie der konfuzianischen Philosophie, auch in ihrer modernen Ausprägung, entsprechen. Der „Lei Feng Spirit“ lässt sich also nicht ohne weiteres exportieren und bleibt ein genuin chinesisches Phänomen.

\section{Der tragische Chor: Das, Volk' als heroisches Subjekt der Geschichte}

Mao Zedong schreibt 1947 über die Härte der militärischen Auseinandersetzung: „Wir müssen unseren Kampfstil voll entwickeln, d.h. mutig kämpfen, keine Opfer scheuen, keine Erschöpfung fürchten und unablässig Kämpfe führen." ${ }^{41}$ Kühnheit, Opferbereitschaft und Selbstüberwindung, kurz: Tapferkeit und Todesverachtung, gelten innerhalb des heroischen Komplexes als komplementär. Die Selbstaufopferung im Dienste einer höheren Idee, welche die Grenzen des Individuellen transzendiert, mithin die willige Hingabe des eigenen Lebens, gehört zu den wesentlichen Merkmalen des Heldentums. ${ }^{42}$

Heroismus wird in der Tradition des Westens als Handlungs- und Lebensoption eines Einzelnen gesehen. Symbolischer Ausdruck hierfür ist die devotio der römischen Feldherren, die den Sieg durch das Opfer ihres eigenen Lebens herbeiführen wollen (vgl. Anm. 2). Der Weihecharakter des Selbstopfers trägt sakralen Charakter - ein Akt herausgehobener Einsamkeit. Gerade dies, die Nähe zu den Göttern, hebt den Heros von der Gesichtslosigkeit der Menge ab. Die Geste ist allerdings voraussetzungsvoll; sie baut auf einer statischen gesellschaftlichen Hierarchie und intakten religiösen Überzeugungen auf. In der säkularisierten Mittelklassegesellschaft abendländischen Zuschnitts wäre eine solche Handlungsweise geradezu abstrus. Für wen oder was sollte man sich opfern? Was wäre absolut anbetungsund dadurch ultimativ verteidigungswürdig? Gerade das Ausbleiben - oder die Unmöglichkeit - einer schlüssigen Antwort bezeichnet die innere Leerstelle, den kulturellen Hohlraum, welche den Westen heute kennzeichnet.

41 Zedong: Worte des Vorsitzenden (Anm. 8), S. 114.

42 Agnes Smedley berichtet von den letzten Worten eines chinesischen Rotarmisten, der von Truppen der Guomintang gefangengenommen und hingerichtet wurde (Smedley: China blutet [Anm. 19], S. 292): „,Ich bin Kommunist!', sagte er stolz. ,Tötet mich, ihr Feinde des Volkes!““ 
Eine völlig diametrale Betrachtungs- und Interpretationsweise des Heroischen ergibt sich aus der modernen chinesischen Geschichte. Akteure der Revolution sind gemäß offizieller chinesischer Lesart vor allem die Massen des Volkes, nicht nur deren Kommandeure. Mao Zedong bemerkt: „Alle Ansichten, in denen eine Überschätzung der Kräfte des Feindes und eine Unterschätzung der Kräfte des Volkes zum Ausdruck kommen, sind falsch. ${ }^{" 43}$ Eine Armee, die aus den Massen des Volkes besteht und zugleich für deren Befreiung kämpft, besitzt eine gewisse revolutionäre Logik, die sich in einer schwer zu erschütternden Zuversicht artikuliert: „Victory will not be easy, but we will fight until victorious. We have our faith - tell your countrymen." ${ }^{44}$ Die gewaltige Emanzipationskraft des Volkes als geschichtliches Subjekt ist unter bestimmten Bedingungen hinreichend für den Sieg, setzt aber beträchtliche individuelle Willenskraft und kollektive Anstrengung voraus: „Wenn es nach unserem Wunsch ginge, würden wir keinen einzigen Tag Krieg führen. Aber wenn uns die Umstände dazu zwingen, können wir bis zum Ende kämpfen. "45 Die geschichtliche Erfahrung einer siegreichen Armee des Volkes ist in Europa auf das revolutionäre Frankreich von 1789 begrenzt; in Russland und China hingegen ist sie jüngeren Datums und politisch noch immer ungebrochen virulent. Nach maoistischem Verständnis ist primär nicht der Einzelne, den es zwar auch benötigt, sondern insbesondere die Masse, die Vielheit, das Volk aktives Subjekt der Geschichte und der entscheidende Faktor gesellschaftlichen Fortschritts. Nichts könnte weiter entfernt sein von den pessimistischen Geschichtskonzeptionen des Westens. ${ }^{46}$ Paradox gesagt: Eine atomisierte Gesellschaft, in der das Individuum die zentrale Figur darstellt, bringt keine Heroen, sondern bestenfalls Lebenskünstler hervor. Eine kollektivistische Gesellschaft, in der die Gemeinschaft als zentraler Bezugspunkt gilt, generiert eine Vielzahl individueller Vorbilder. Diese können aber nur aus der Mitte der Gesellschaft, aus der ,Mitte des Volkes‘ kommen - als Arbeiter und Bauern. Aus dem namenlosen Chor tritt der geschichtliche Protagonist.

Die Archaik der Arbeitswelt und die ästhetisch-ideologische Überhöhung des körperlich arbeitenden Menschen bilden eine der letzten modernen Inseln des Heroismus. Als Beispiel dient der Bergbau, noch immer eines der riskantesten Berufsfelder. Während in Deutschland die letzte Kohlemine Ende 2018 schloss, hat der Bergbau in Süd- und Osteuropa, ebenso wie in China, noch eine große wirtschaftliche Bedeutung. Die spanische Ethnologin Latorre Pallares zitiert einen spanischen Bergarbeiter:

43 Zedong: Worte des Vorsitzenden (Anm. 8), S. 102.

44 So der Kommandeur General Chung Yi in einem Interview mit Agnes Smedley (Smedley: Battle Hymn of China [Anm. 23], S. 363), sechs Jahre vor dem Sieg der revolutionären Truppen.

45 Zedong: Worte des Vorsitzenden (Anm. 8), S. 100.

46 Vgl. z. B. Reinhart Kosellecks klassische Studie Kritik und Krise von 1959. 
Der Bergbau, auch wenn er noch so fürchterlich und düster erscheint, hat auch seine Poesie; eine harte und raue Poesie, wenn man so will, aber letztendlich doch Poesie. Sie verherrlicht die Arbeit, erhöht das Heldentum und rühmt die Figur des Bergarbeiters, die Tugend der Kameradschaft und Solidarität [...]. Es ist Opferbereitschaft in der stummen und konstruktiven Arbeit dieser ganzen Legion von Männern, Titanen des Erdinnern [...]. Ist es nicht heroisch, das Gedicht, das der Bergmann schreibt, wenn er oft sein eigenes Leben aufs Spiel setzt, um das seines Kumpels zu retten? ${ }^{47}$

In der aussterbenden sozialen Spezies des Arbeiters manifestiert sich eine weitere Variante heroischer Figurationen, die heute ebenso symbolträchtig wie gesellschaftlich wirkungslos sind. Als Mann des Volkes repräsentiert der Arbeiter den namenlosen Chor der Geschichte und leiht ihm für einen kurzen Moment seine Stimme: „The eternal truth that is beyond all social systems - the equality of men before eternity and their equal rights in this life to all that is good." ${ }^{8}$ Die proletarische Version des Helden als Arbeiter ${ }^{49}$ vereint in sich, wie die Figur des Helden als Krieger, existentielles Ethos und tödliches Pathos. Die Risiken, welche beide eingehen, sind real, notwendig und unausweichlich. In dieser harten Konfrontation mit der Wirklichkeit - widerständiges Material oder bewaffneter Feind - bezeugen sie ihren eigenen absoluten Standpunkt, den sie um den Preis ihres Lebens behaupten. Sie folgen darin einer Konsequenz, die sich mit den Maßstäben der bürgerlichen Gesellschaft schlechterdings nicht ermessen lässt. Der namenlose Held in der Geschichte, ob Prolet oder Krieger, ist der natürliche Gegenspieler des Bürgers. Dieser ist geradezu sein ärgster Feind, denn in der bürgerlichen Welt, die Konflikte grundsätzlich nicht offen ausagiert, ist der Held in seiner konfrontativen Bedingungslosigkeit zum Aussterben oder zum Gefängnis verurteilt.

\section{Schlussbetrachtung}

Die Differenzen im Begriff des Heroischen zwischen China und Deutschland sind immens, wenn nicht sogar unüberbrückbar. Die historischen und moralischen Vorzeichen des Heldentums und seiner Deutung sind in beiden Ländern geradezu spiegelverkehrt. Der chinesische Held, exemplarisch verkörpert in der Person Lei Fengs, vertritt den siegreichen Kommunismus und ist eine Gestalt des Triumphes. Von dieser erhöhten Position wirkt er als medial inszeniertes gesellschaftliches Vorbild und Orientierungsmuster. Die kollektivistische Deutungsdimension, im Konfuzianismus bereits angelegt, ist ebenfalls spezifisch für seine Rezeptionsgeschichte seit den 1960er Jahren. Der moderne Held chinesischer Prägung ist, kurz gesagt, eine systemaffirmative Figur. In Deutschland dagegen ist die gesellschaftliche Rolle

47 Andrés Fernández, zit. nach: Patricia Latorre Pallares: Der Kumpel - „Held der Arbeit“ und ,geborener Rebell“? Kultureller Machtkampf um die Arbeit im asturischen Kohlerevier, Frankfurt am Main 2001, S. 18.

48 Brief aus Salzburg (1926) von Agnes Smedley an ihre Freundin Florence Lennon. Zit. nach: MacKinnon: The Life and Times of an American Radical (Anm. 12), S. 108.

49 Vgl. Jünger: Der Arbeiter (Anm. 16). 
des Helden als Krieger seit langem unbesetzt. Sie gehört zu der verbrannten geschichtlichen Welt, welche zwei verlorene Weltkriege hinterlassen haben. Repräsentativ ist daher nicht der Sieger, sondern der Gescheiterte. Lehmbrucks eindrückliche Plastik Der Gestürzte (1918) kann als deutsches Epochengleichnis gesehen werden. Der Held wird historisch unter dem Vorzeichen des totalen Krieges und der totalen Niederlage gesehen und ist eine Gestalt der Vergeblichkeit. Diese Betrachtung des heroischen Phänomens aus einer Perspektive der Niederlage und der Opposition ist sicherlich eine deutsche Eigenart. Frankreich oder England teilen diese keineswegs. ${ }^{50}$ Helden sind hierzulande nicht die tatendurstigen Konformisten, sondern die einsamen Verweigerer staatlicher und politischer Macht. Letzteres wäre in China wiederum schwer vorstellbar. Als geradezu unvereinbar erweist sich das individualistische Lebens- und Leistungsideal des Westens mit der konfuzianischen und kollektivistischen Tradition. Russland steht hier China nicht nur geographisch, sondern auch ideologisch wesentlich näher. ${ }^{51}$

Die Unterschiede in Verständnis und Praxis des heroischen Komplexes bündeln wie in einem Brennglas die Unterschiede zwischen der europäischen, exemplarisch der deutschen, und der chinesischen Kultur. Die jeweilige geschichtliche Logik dahinter lässt sich benennen, darstellen und am konkreten Beispiel veranschaulichen. Dies bedeutet jedoch nicht, dass sich beide Kulturen innerlich annähern oder ihre Mentalitäten und Lebensmodelle aneinander angleichen würden. Die Feststellung einer prinzipiellen Differenz der chinesischen Kultur gegenüber jeder anderen ist die wahre Lektion Lei Fengs.

50 So würdigte der französische Staatspräsident Emmanuel Macron den Polizisten Arnaud Beltrame, der bei einer terroristischen Geiselnahme ums Leben kam, mit den Worten: „Alors que le nom de son assassin déjà sombrait dans l'oubli, le nom d'Arnaud Beltrame devenait celui de l'héroïsme français, porteur de cet esprit de résistance qui est l'affirmation suprême de ce que nous sommes, de ce pour quoi la France toujours s'est battue, de Jeanne d'Arc au Général De Gaulle: son indépendance, sa liberté, son esprit de tolérance et de paix contre toutes les hégémonies, tous les fanatismes, tous les totalitarismes." Emmanuel Macron: Discours du président de la République en hommage au lieutenant-colonel Arnaud Beltrame, 28. März 2018, en-marche.fr/articles/discours/discours-du-president-de-la-republique-enhommage-au-lieutenant-colonel-arnaud-beltrame, 10. Dezember 2018 (Hervorh. d. Verf.). Diese selbstverständliche Beschwörung einer ungebrochenen heroischen Traditionslinie vom 15. bis ins 20. Jahrhundert wäre in Deutschland schlicht undenkbar.

$51 \mathrm{Zu}$ sino-russischen Gemeinsamkeiten und Unterschieden vgl. die ausführliche Studie von Parag Khanna: The Future is Asian, New York 2019, S. 19-20, S. 82-85. 
